
identification of local adaptation actions

\title{
Vulnerabilidad de los manglares en la reserva de la biósfera el vizcaíno, e identificación de acciones de adaptación local
}

Karina Alely Bautista-González ${ }^{1}$

\section{Resumen}

Este trabajo constituye el primer análisis de la vulnerabilidad de los manglares y de los medios de vida llevada a cabo en seis comunidades costeras de Baja California Sur, México. Este estudio se hizo desde la perspectiva social, tomando en cuenta la visión de las personas, quienes toman decisiones para impulsar acciones que ayuden a disminuir la vulnerabilidad de manera más local y fortalecer sus capacidades de adaptación. Así mismo, se analizó la capacidad adaptativa que tienen estas comunidades y cómo están afrontando estos cambios. Se encontró que la capacidad adaptativa de las comunidades es diferente debido a las acciones que están realizando, a la forma de organizarse y la diversificación de sus medios de vida. Por último, se identificaron de manera participativa acciones para la adaptación local, para disminuir la vulnerabilidad de los manglares y de los medios de vida.

Palabras claves: cambio climático, capacidad de adaptación, medios de vida, vulnerabilidad.

\section{Abstract}

This work constitutes the first analysis of mangrove vulnerability and livelihoods carried out in six coastal communities of Baja California Sur, Mexico. This study was done from the social perspective, taking into account the vision of the people involved in decision making to promote actions that help to reduce vulnerability locally and strengthen their adaptive capacities. Likewise, the adaptive capacity of these communities and how they are coping with these changes were

\footnotetext{
${ }^{1}$ Centro Agronómico Tropical de Investigación y Enseñanza (CATIE), Turrialba, Cartago, Costa Rica.
} Kbautista@catie.ac.cr; kabg18@gmail.com 
analyzed. It was found that the adaptive capacity of the communities was different because of the actions they were doing, the way they were organized and the diversification of their livelihoods. Finally, actions for local adaptation were identified in a participatory manner, to reduce mangrove vulnerability and livelihoods.

Keywords: Adaptive capacity, climate change, livelihood, vulnerability.

\section{Introducción}

El estado de Baja California Sur es uno de los estados con mayor crecimiento económico y demográfico en México y, por su ubicación geográfica, una de las regiones más amenazadas en lo que respecta a la conservación de su riqueza natural. Los manglares de esta región son particularmente vulnerables porque están en el norte en el límite de su distribución de la costa del Pacífico. Son importantes porque de su estabilidad dependen las principales actividades económicas de la población local (p.ej., la pesca y el turismo).

Los manglares son hábitats que estabilizan y protegen los paisajes costeros, contribuyen al mantenimiento de la calidad de agua costera y funcionan como hábitat principal y de reproducción para muchas especies con importancia comercial (mamíferos, aves, reptiles y peces). Posiblemente son los ecosistemas costeros más afectados en el mundo por el aumento en el nivel del mar, la temperatura y la mayor frecuencia e intensidad de huracanes y tormentas (Cifuentes-Jara 2010).

Para Sandoval-Castro et al. (2014) Los manglares son ecosistemas tropicales altamente productivos que soportan numerosas cadenas alimentarias en la zona costera y en los ecosistemas vecinos. La presencia de manglares tiene un fuerte efecto en los rendimientos de la pesca, ya que proporcionan alimento a los sistemas costa afuera como fuente de carbono. Los manglares también proporcionan protección contra la erosión de las costas al reducir la energía de las olas, bloqueando el sedimento con sus raíces y promoviendo la sedimentación. Por lo tanto, comprender la respuesta de las especies de manglares al cambio climático es de suma importancia para el manejo de los recursos costeros.

El cambio climático global está condicionando una nueva visión, no solo ecológica en la apreciación del funcionamiento de los ecosistemas costeros, sino también socio económica 
replanteando consideraciones para el manejo costero integrado en el Golfo de México (Day et al., 2013a; Yáñez-Arancibia et al., 2013a, 2013b citado por Yañez -Arancibia et al, 2014).

\section{La importancia de los manglares y su distribución}

Hay evidencia de que el cambio climático, en particular el aumento del nivel del mar, tendrá un impacto significativo en los ecosistemas costeros, específicamente aquellos que ya están amenazados por otras perturbaciones antropogénicas, y los ecosistemas costeros mexicanos no son una excepción. Sin embargo, su evaluación de la resiliencia a futuros cambios climáticos a menudo se ve obstaculizada por la falta de datos e información apropiados. Esto dificulta la implementación de estrategias efectivas de conservación en estos entornos vulnerables (Hernandez-Montilla, et al, 2016).

La distribución global del manglar está influenciada principalmente por la temperatura, restringiendo las especies a las latitudes tropicales y subtropicales cálidas. Se han planteado hipótesis sobre los fenómenos de frío extremo para explicar las transformaciones del rango y las extinciones regionales severas y los límites latitudinales de la distribución del manglar (SandovalCastro, 2014).

Los manglares constituyen un importante recurso forestal en toda la banda intertropical del planeta (ca.24, 720 km2, pero también se han señalado 1590 ha). Los componentes de biodiversidad de estos ecosistemas son únicos debido a que incluyen nichos estructurales, son fronteras a nivel de eco-regiones, y actúan como refugio para numerosa fauna y especies microbianas y proveen una diversidad de bienes y servicios (Yáñez-Arancibia et al. 1998). En este estudio se logró analizar los cambios en las variables climáticas y no climáticas que están afectando a estos ecosistemas de la Laguna de San Ignacio (LSI). La sinergia entre las mismas, podría estar ocasionando afectaciones en la salud de los manglares y limitando su desarrollo. Asimismo ayudar en la toma decisiones de los manejadores del territorio y redireccionar las acciones, proyectos e iniciativas locales que se estén llevando a cabo o que recién inicien para su fortalecimiento y sustentabilidad, que permitan disminuir la vulnerabilidad y mejorar la capacidad de adaptación.

Variables que podrían afectar la distribución y salud de los manglares, como el aumento del nivel del mar, del aire y de la temperatura superficial del mar que muestran tendencias a aumentar bajo diferentes proyecciones de clima futuro. Las personas perciben los cambios en la temperatura del 
agua, del aire y el efecto de El Niño, así como cambios en los oleajes y mareas, lo que coincide con resultados de un reciente informe sobre el plan estatal de acción al cambio climático (PEACC-BCS, 2012). Esto afectaría considerablemente los principales medios de vida de las comunidades como la pesca, acuacultura y el turismo, impactando negativamente el desarrollo de estas comunidades costeras. El tipo y magnitud de los impactos dependerá de las características ambientales, sociales y económicas de cada comunidad o región e influirá sobre el grado de afectación a los manglares y a las comunidades. Hay diferentes maneras de estimar la vulnerabilidad, según la perspectiva científica y social.

Este estudio está enfocado en el análisis de la vulnerabilidad ante el cambio climático de los manglares de la Laguna de San Ignacio (LSI), en la costa occidental de La Baja California Sur (BCS), noroeste de México, y en la identificación de acciones locales para la adaptación de las comunidades aledañas.

Se espera que este análisis sirva como una herramienta en la gestión y planificación de proyectos similares, que estén enfocados a las acciones de conservación, uso y manejo sostenible de los manglares y a fortalecer capacidad adaptativa de comunidades costeras.

\section{Área de estudio}

\section{Manglares de la Laguna de San Ignacio y su gente}

La estabilidad de los manglares (Rhizophora mangle y Laguncularia recemosa) de la LSI, podría estar amenazada por los efectos del cambio climático. Igual que en otras partes del planeta, los manglares en BCS han sido afectados principalmente por disturbios naturales, incluyendo eventos meteorológicos intensos como vientos fuertes, avenidas extraordinarias de aguas pluviales y diversos efectos locales derivados del cambio climático global, además de cambios físicos del suelo (Emanuel 2005, McGranahan et al. 2007, Bender citado por Mendoza et al. 2011).

Las comunidades aledañas a la LSI: El Cardón, El Ejido Luis Echeverría, El Dátil, El Delgadito, Punta Abreojos y La Bocana (Figura 1), dependen en gran medida del aprovechamiento de bienes y servicios provenientes de los manglares: pesca y turismo, son comunidades que, directa e indirectamente, tienen relación dependiente con el ecosistema. Por esa dependencia de los medios de vida de las comunidades costeras en estos ecosistemas, es necesario implementar acciones para: 
a.- Identificar los impactos potenciales del cambio climático en los manglares así como las principales actividades humanas que pueden acentuarlos.

b.- Fortalecer la capacidad adaptativa local de las comunidades humanas en la costa de la BCS ante los cambios y los eventos extremos que inevitablemente puedan ocurrir.

c.- Reducir la sensibilidad de las infraestructuras costeras.

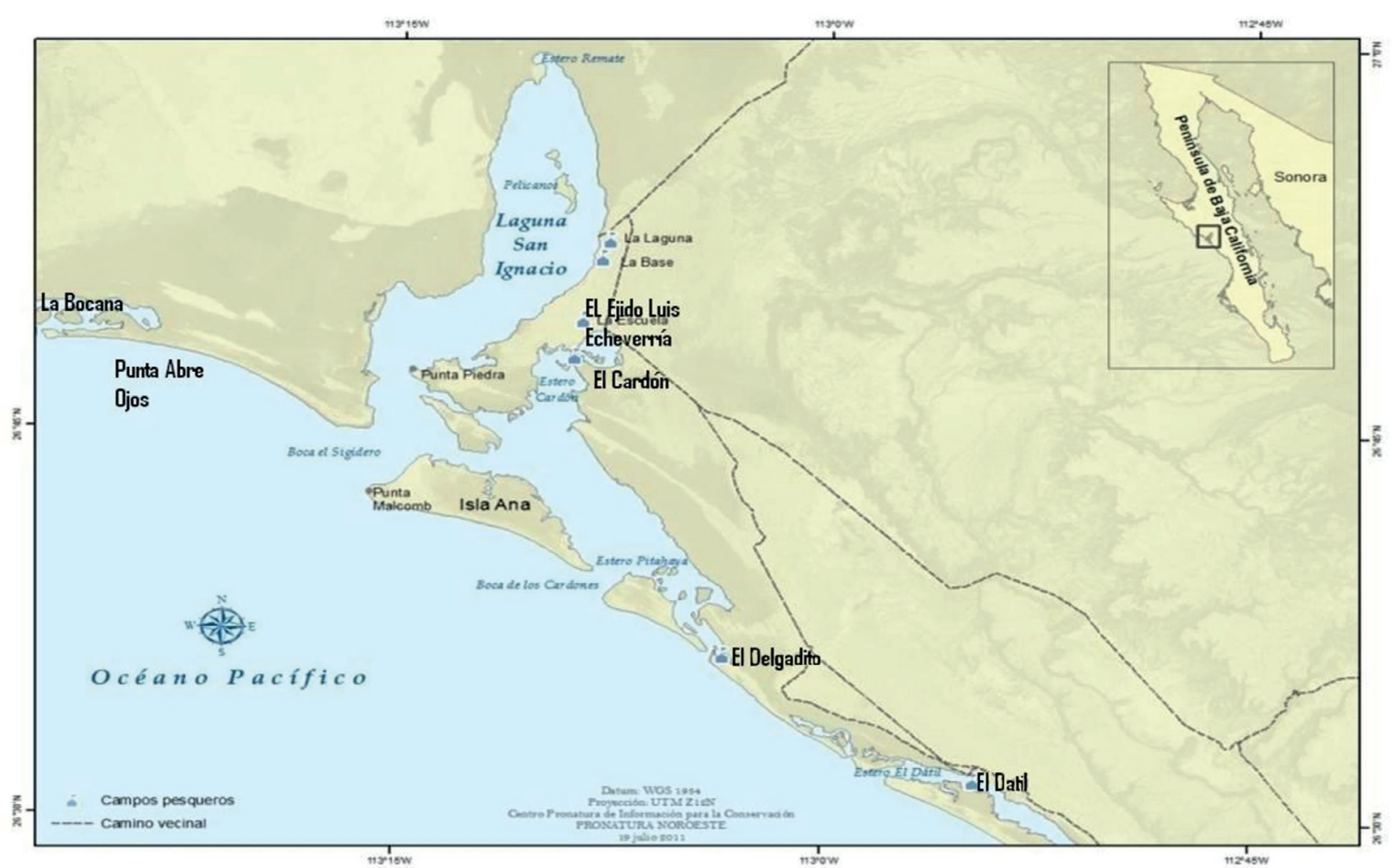

Figura 1. Ubicación de las comunidades cercanas a la Laguna de San Ignacio, Baja California Sur, México. Fuente: Pronatura A.C. (2011)

Cambios proyectados en la temperatura superficial del mar, la temperatura del aire y el aumento del nivel del mar de Baja California Sur

Las variables relacionadas con el establecimiento y la estabilidad ecológica de los manglares son: la temperatura superficial del mar (TSM), la temperatura del aire (TA) y nivel medio del mar (ANM). Los cambios han aumentado en estas variables podrían estar afectando la salud de los manglares.

Para estimar el incremento de la TSM para BCS, se consideraron proyecciones de los periodos del clima del tiempo presente (1979-2003), y clima del tiempo futuro (2075-2099), realizadas con 
escenarios A1B. Las tendencias para todo BCS señalan un aumento de $2{ }^{\circ} \mathrm{C}$ (PEACC-BCS 2012) entre ahora y finales del siglo. Las proyecciones de la TA para los periodos 2010-2029 y 2080-20, considerando los escenarios de emisiones A1y B1, señalan un aumento de $1^{\circ} \mathrm{C}$ a $4.5^{\circ} \mathrm{C}$ entre ahora y finales del siglo (Figura 2).

Para BCS se espera un cambio en el nivel medio del nivel del mar de -3.45 y $0.52 \mathrm{~mm}$ al año. Para la LSI la tendencia de aumento es mayor, de 2 a $2.5 \mathrm{~mm}$ anuales entre 1992 y 2012 (AVISO 2013; Figura 3).

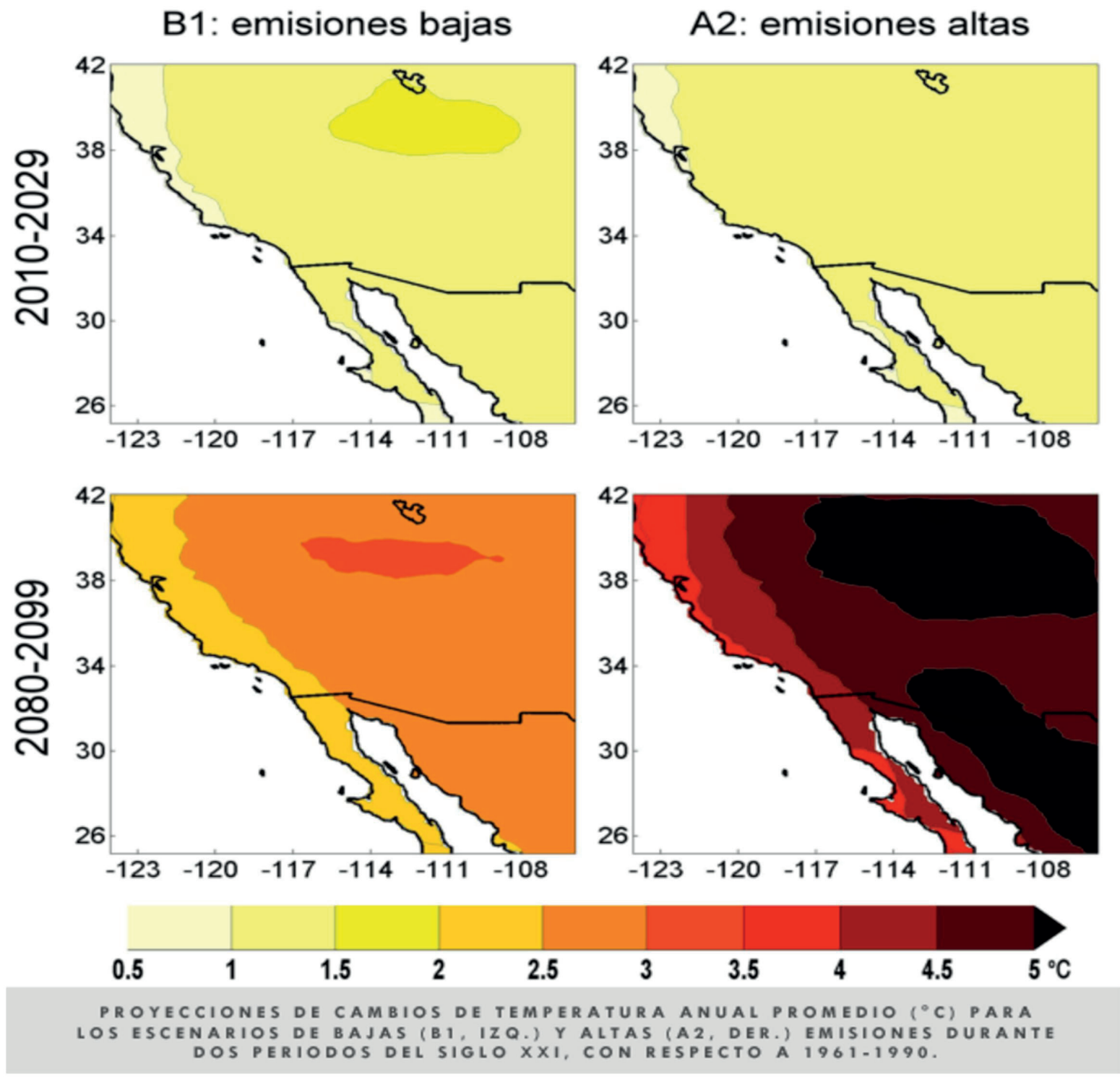

Figura 2. Cambios de la temperatura del aire proyectados para los periodos 2010 - 2029 y 2080 - 2099, según los escenarios de emisiones B1 y A2 para la Baja California Sur, México 


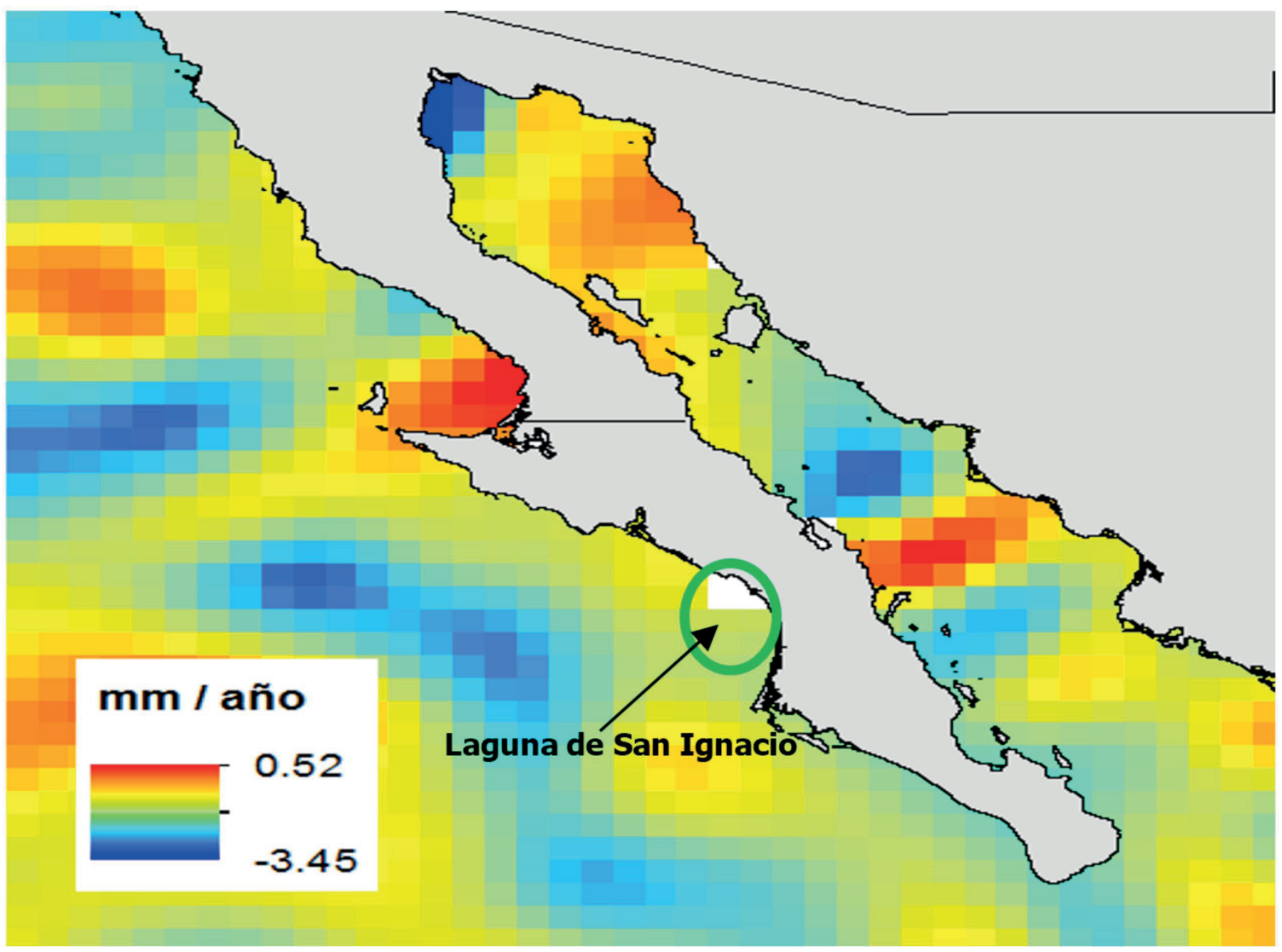

Figura 3. Tendencia del aumento del nivel del mar en la Laguna de San Ignacio, Baja California Sur, México entre octubre 1992 y diciembre 2012. Fuente: AVISO

\section{Metodología}

Análisis de vulnerabilidad e identificación de acciones para la adaptación local

La metodología para la obtención y análisis de la información se basó en el marco de los capitales de la comunidad (MCC) y los medios de vida sostenibles (MVS).

Se realizó una revisión de información secundaria disponible relacionada al área de trabajo. En esta etapa de diagnóstico y contextualización, también se realizó un mapeo de actores mediante reuniones, entrevistas y charlas formales y no formales. Se consideraron a los actores como: organizaciones, instituciones y personas que trabajan en el área de estudio y que tienen relación con los manglares.

Métodos utilizados en la obtención de la información para los análisis de vulnerabilidad y la identificación de acciones para la adaptación local

Grupos focales: Se realizaron diez reuniones en seis comunidades que forman parte del área 
protegida en la Laguna de San Ignacio. En las reuniones participaron los actores identificados (un promedio de 6 participantes por reunión o grupo focal) que brindarían información relacionada a la exposición, sensibilidad y capacidad adaptativa. También mediante estos grupos focales se identificaron las acciones locales para la adaptación. En total participaron 60 personas en los grupos focales, fueron pobladores de las comunidades que tuvieran una relación con el tema, vinculados a diversas actividades: pesca, acuacultura, turismo. Las personas que participaron fueron socios de las cooperativas pesqueras, ejidatarios, personas involucradas en los proyectos de vigilancia comunitaria y proyectos de conservación. Los grupos focales se realizaron para profundizar y generar discusión sobre los temas y visibilizar aspectos de la situación actual de los manglares, de los medios de vida de las comunidades y sobre los recursos disponibles.

Recorridos: Se realizaron seis recorridos, uno en cada comunidad, organizados con personas de cada comunidad (expertos locales), para conocer la dinámica de las comunidades. Los recorridos realizados en el manglar sirvieron como complemento a la información generada en los grupos focales. Permitieron conocer el estado actual de los manglares, problemas que las personas mostraron en los recorridos y lo que creen podrían estar ocasionando esas afectaciones. Así mismo, sirvió para retroalimentar la información facilitada por los pobladores de las comunidades y por medio de la guía de preguntas utilizadas en los grupos focales se tomaron notas de campo.

Entrevistas: Se llevaron a cabo diez entrevistas a los actores claves en las comunidades y representantes de organizaciones, instituciones y tomadores de decisión. Las preguntas utilizadas para estas entrevistas fueron las mismas del protocolo para los grupos focales. Estas entrevistas sirvieron para complementar información de los grupos focales y también para triangular la información.

Los instrumentos utilizados incluyeron preguntas específicas relacionadas con los medios de vida y los capitales de la comunidad, exposición y sensibilidad al cambio climático y capacidad adaptativa. Se aplicaron en las seis comunidades aledañas a la LSI: El Cardón, El Delgadito, El Dátil, Ejido Luis Echeverría y Punta Abre Ojos y La Bocana. Se involucraron actores claves institucionales: la dirección de la Reserva de la Biosfera del Vizcaíno (REBIVI), el municipio de Mulegé , el representante de pesca y acuacultura, el presidente de la asociación de silvicultores 
de Mulegé y el presidente del Consejo Asesor de la REBIVI. En todo el proceso participaron 70 personas: 60 en los grupos focales y 10 en las entrevistas.

La información se organizó por comunidades, elementos del análisis de vulnerabilidad (exposición, sensibilidad y capacidad adaptativa) y los medios de vida de las comunidades.

Análisis de la información: Se realizó una síntesis de la información mediante cuadros a fin de resumir lo más relevante sobre las percepciones de exposición y sensibilidad, que es necesario para determinar el impacto potencial. La capacidad adaptativa de las comunidades aledañas a la LSI se analizó a través de las fortalezas y debilidades de las comunidades, los capitales de la comunidad y los medios de vida. Por último, las propuestas de acciones para la adaptación local se organizaron por temas enfocados a fortalecer las capacidades locales vinculadas a sus capitales y medios de vida.

\section{Resultados}

Impacto potencial del cambio climático en los manglares y medios de vida según la percepción de los pobladores de las comunidades aledañas a la Laguna San Ignacio

Percepción de cambios en el clima y de procesos no climáticos

Los pobladores de todas las comunidades que participaron en las entrevistas y grupos focales consideran que sí hay cambios en las variables climáticas analizadas. Todas las personas involucradas en este trabajo coinciden en cuanto al aumento de la temperatura del aire y los cambios en las lluvias. Consideran que éstas ya no son tan estacionales como antes: ahora solo llueve en temporadas de huracanes. Las temperaturas son más extremas: más calor en verano y menores temperaturas en invierno.

Las personas de las diferentes comunidades tienen diferentes percepciones con relación a los cambios observados en oleajes y las mareas. La ubicación de las zonas pobladas respecto a la boca del estero y a la orilla define su diferente exposición a las mareas y oleajes. Punta Abre Ojos y La Bocana están más cerca de las bocas del estero pero sus construcciones están en zonas protegidas de la marea, excepto la zona turística del estero El Coyote (de Punta Abre Ojos). En La Bocana no han tenido problemas con inundaciones. El Cardón, Luis Echeverría, El Delgadito y El Dátil están en el interior del estero pero sus construcciones están en zonas más bajas. Las áreas afectadas por 
las mareas más altas son las casas de El Cardón y un campo turístico en Luis Echeverría. Algunas personas perciben el inicio de estos cambios 20 años atrás.

El efecto del fenómeno El Niño (ENOS) es otro cambio que no todos los pobladores perciben de igual manera sobre sus medios de vida. Sus efectos son más evidentes para los pobladores de las comunidades que producen ostión y/o extraen almeja "pata de mula" en el estero. Ellos relacionan el efecto del ENOS con el aumento de la temperatura del agua, cambios en la circulación de las mareas y la aparición de mareas rojas. La percepción de los efectos del ENOS en el Ejido Luis Echeverría, Punta Abre Ojos-Estero El Coyote y La Bocana es menor porque están en zonas más abiertas.

A partir del análisis de la exposición, se determinó que no solo el clima está afectando a los manglares y medios de vida, sino también la presión de la contaminación y las prácticas de pesca no adecuadas. En las comunidades El Cardón, El Dátil, El Delgadito y El Ejido Luis Echeverría ha aumentado el impacto por esos factores antes mencionados.

Sin embargo, en las comunidades de Punta Abreojos y La Bocana han logrado disminuir la contaminación y las malas prácticas de pesca. Esto ha favorecido a los manglares para su desarrollo y conservación. Por medio de las cooperativas de producción pesquera El Progreso, La Bocana y Punta Abreojos tienen una vigilancia permanente. A través de un responsable con la facultad de sancionar y promover el orden. Siendo la autoridad local importante en el cumplimiento de los acuerdos internos para la pesca y la protección del estero, ha permitido que sus medios de vida mejoren para seguir aprovechando mejor sus recursos pesqueros.

En resumen, todas las comunidades perciben que están expuestas al aumento de las mareas y oleajes, porque se encuentran en zonas bajas y más abiertas a las mareas. Las viviendas de El Cardón son las que más se inundan por estar en una zona de alto riesgo. El campo turístico Kuyima que forma parte del Ejido Luis Echeverría, también tuvieron problemas con inundación, pero sin perdidas en la infraestructura. El campo turístico René del estero el Coyote (Soc. Coop. Punta Abre Ojos) también se les inundaron las cabañas. La Bocana no mencionó problemas con inundación.

El calentamiento del agua con la asociación del ENOS, les afecta más a las comunidades del Cardón y al Ejido Luis Echeverría porque cultivan ostión y El Dátil, por la extracción de la almeja de "pata de mula". No afecta a El Delgadito porque la gente de esta comunidad pesca a mar 
abierto. En Punta Abre Ojos y La Bocana cultivan ostión, pero el aumento de temperatura no ha sido tan alto porque están en una zona más abierta donde la circulación del viento es mayor y también es una zona más que las otras comunidades antes mencionadas fría.

Hay que considerar que en El Dátil y El Cardón hay más problemas con la contaminación y mayor incidencia de malas prácticas de pesca. De ahí le siguen El Delgadito y El Ejido Luis Echeverría. La Bocana y Punta Abre Ojos, por medio de sus cooperativas, buscan mitigar esos problemas, fomentando la vigilancia de los recursos pesqueros y del estero.

Cuadro I. Afectaciones ocasionadas por variables climáticas y no climáticas en comunidades aledañas a la Laguna de San Ignacio, Baja California Sur, México

\begin{tabular}{|c|c|c|c|c|c|c|}
\hline $\begin{array}{l}\text { Variables climáticas y no } \\
\text { climáticas }\end{array}$ & El Cardón & El Delgadito & El Dátil & $\begin{array}{l}\text { Ejido Luis } \\
\text { Echeverría }\end{array}$ & Punta Abre Ojos & La Bocana \\
\hline \multicolumn{7}{|l|}{ Mareas y oleajes } \\
\hline \multicolumn{7}{|l|}{ Niño } \\
\hline \multicolumn{7}{|l|}{ Contaminación } \\
\hline Malas prácticas de pesca & & & & & & \\
\hline
\end{tabular}

Percepción del impacto del cambio climático y procesos no climáticos en los manglares y medios de vida de las comunidades

Los cambios en la temperatura del agua y aire, y el ENOS, en combinación con la contaminación y las malas prácticas de pesca (en algunos casos), han afectado negativamente los manglares de todas las comunidades del área de estudio. Los cambios que más los afectan son los oleajes y marejadas mayores, el aumento de la temperatura del agua y del aire. Los manglares son sensibles a estos cambios y comienzan a modificar su distribución y extensión.

Los manglares más afectados por los cambios en el clima y la sinergia causada por la contaminación y las malas prácticas de pesca, se encuentran en las comunidades de El Cardón, El Dátil, Ejido Luis Echeverría y El Delgadito. Estos manglares se encuentran en algunas partes secos, enterrados, arrancados y contaminados por desechos sólidos (bolsas de plástico, botellas de plástico y desechos de pescado y conchas de almeja). El mangle más afectado por estos cambios ha sido el blanco (Laguncularia racemosa).

La mayor frecuencia y altura de las marejadas es otro factor que ejerce presión sobre la actividad pesquera porque hace que los pescadores de las comunidades pierdan días de trabajo. También 
provoca que tengan pérdidas económicas, principalmente durante las temporadas de pesca de especies con alto valor comercial.

Se han observado cambios en la distribución de las principales especies comerciales, al parecer por el calentamiento del agua. El Cardón, El Delgadito, Dátil y El Ejido Luis Echeverría son las comunidades más afectadas. Punta Abre Ojos y La Bocana son menos afectadas por estar en zonas más frías y la población percibe que cerca de la boca el agua no se calienta mucho. Ha disminuido el volumen de captura y extracción de almejas, peces, langostas y abulón También las temporadas de apertura de las vedas se han atrasado o viceversa.

La industria pesquera de las sociedades cooperativas de las comunidades de Punta Abre Ojos y La Bocana, comentan que les ha afectado la disminución de los volúmenes de captura del producto pesquero, para procesar y empaca, limitándoles cumplir con el abastecimiento del mercado.

El Cardón, El Ejido Luis Echeverría y Punta Abre Ojos han tenido pérdidas en el cultivo de ostión por el aumento de temperatura asociada al ENOS.

La extracción de la "pata de mula" ha tenido un impacto debido al aumento de la temperatura asociada al ENOS; su aprovechamiento por parte de las familias que desarrollan esta actividad se ha limitado. Los más afectados son la comunidad de El Dátil, ya que forma parte de sus principales medios de vida.

La actividad turística se ha visto afectada principalmente por el aumento de la marea, que ha provocado inundaciones en la comunidad El Cardón y campos turísticos del Ejido Luis Echeverría (campo Kuyima) y Punta Abre Ojos (campo René-Estero El Coyote). Además, en la LSI, consideran que las ballenas comienzan a retrasarse en la temporada o adelantarse en su migración. Las aves migratorias, otro atractivo turístico, también han adelantado sus fechas de llegada a la zona.

Por último, en la comunidad El Delgadito las afectaciones fueron el limitado acceso en vía terrestre para movilizarse a otras comunidades para la compra de alimentos y agua.

En resumen, los manglares más sensibles se encuentran en la comunidad El Dátil. Esto se debe a la contaminación y a las malas prácticas de pesca. Aunado a lo anterior, los eventos climáticos extremos, relacionados como impactos del cambio climático han aumentado su vulnerabilidad. Los medios de vida más sensibles son la pesca, la acuacultura y la extracción de la almeja "pata de mula". Porque son más sensibles a cambios en las temperaturas en el agua y el aire, sufren de la influencia del ENOS. Las comunidades que sienten más estos cambios son El Cardón, El Dátil, 
Delgadito y Ejido Luis Echeverría, porque se ubican dentro de la LSI y es la zona donde se calienta más el agua. En cambio, en Punta Abre Ojos y La Bocana el impacto observado es menor porque se ubican en una zona más abierta en la boca y hay más circulación del agua (Cuadro II).

Cuadro II. Sensibilidad de los medios de vida de las comunidades a los cambios en variables ambientales, Laguna de San Ignacio, Baja California Sur, México

\begin{tabular}{|c|c|c|c|c|c|c|}
\hline Medios de vida & El Cardón & Delgadito & Dátil & Luis Echeverría & Punta Abre Ojos & La Bocana \\
\hline \multicolumn{7}{|l|}{ Manglares } \\
\hline \multicolumn{7}{|l|}{ Pesca } \\
\hline \multicolumn{7}{|l|}{ Acuacultura } \\
\hline $\begin{array}{l}\text { Extracción de } \\
\text { almeja }\end{array}$ & & & & & & \\
\hline
\end{tabular}

\section{Capacidad adaptativa}

\section{Condiciones para la adaptación de las comunidades}

La industria pesquera de las sociedades cooperativas de las comunidades de Punta Abreojos y La Bocana, comentan que han tenido una disminución en los volúmenes de capturas del producto pesquero, para procesar y empaca, limitándoles cumplir con el abastecimiento del mercado.

El Cardón, El Ejido Luis Echeverría y Punta Abre Ojos han tenido pérdidas en el cultivo de ostión por el aumento de temperatura asociada al ENOS.

La extracción de la "pata de mula" ha tenido un impacto negativo en la población debido al aumento de la temperatura asociada al ENOS; su aprovechamiento es muy importante por parte de las familias que desarrollan esta actividad más la sinergia con la sobreexplotación, esto ha limitado su desarrollo y aprovechamiento. Los más afectados son la comunidad de El Dátil, ya que forma parte de sus principales medios de vida.

La actividad turística se ha visto afectada principalmente un cambio en el comportamiento del nivel de las mareas, que ha provocado inundaciones en la comunidad El Cardón y campos turísticos del Ejido Luis Echeverría (campo Kuyima) y en Punta Abreojos (campo René-Estero El Coyote). Además, en la LSI, consideran que las ballenas comienzan a retrasarse en la temporada o adelantarse en su migración. Las aves migratorias, otro atractivo turístico, también han adelantado sus fechas de llegada a la zona. 
Por último, en la comunidad El Delgadito, las afectaciones fueron, el limitado acceso en vía terrestre para movilizarse a otras comunidades para la compra de alimentos y agua.

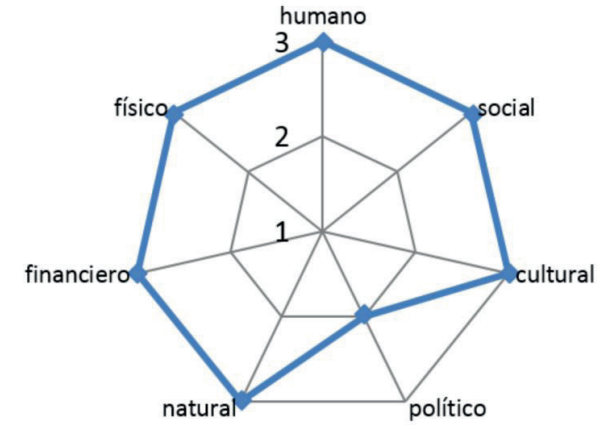

A

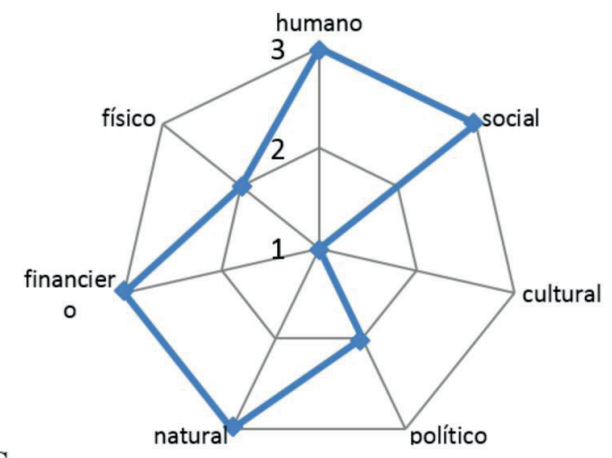

C

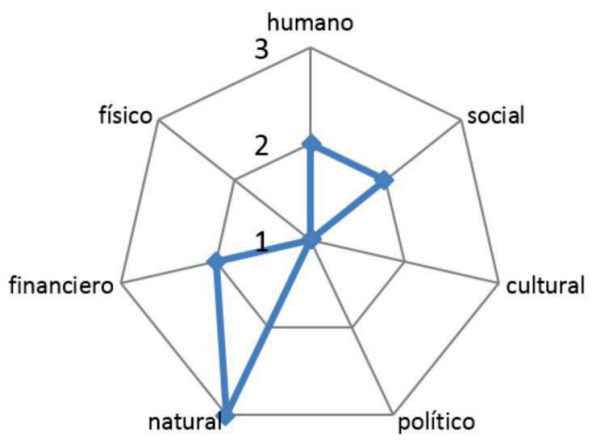

$\mathbf{E}$

Figura 4. Estado actual en los capitales de la comunidad de A) Punta Abre Ojos, B) La Bocana, C) Ejido Luis Echeverría, D) El Delgadito, E) El Cardón y F) El Dátil, Laguna de San Ignacio, Baja California Sur, México 
Cuadro III. Análisis de la capacidad para ejecutar acciones de adaptación de las comunidades aledañas a la Laguna de San Ignacio, Baja California Sur, México

\begin{tabular}{|c|c|c|c|c|c|c|c|}
\hline Capitales & Comunidades & El Cardón & $\begin{array}{l}\text { El } \\
\text { Delgadito }\end{array}$ & El Dátil & $\begin{array}{l}\text { El Ejido Luis } \\
\text { Echeverría }\end{array}$ & $\begin{array}{l}\text { Punta Abre } \\
\text { Ojos }\end{array}$ & La Bocana \\
\hline \multirow{2}{*}{ Natural } & Cambios en el clima & \multicolumn{6}{|c|}{$\begin{array}{l}\text { Tendencia de aumento de la TSM de } 20-30^{\circ} \mathrm{C} \text { como promedio donde los incrementos del } \\
\text { orden de } 2^{\circ} \mathrm{C} \\
\text { En el aumento del nivel del mar indican un aumento de } 2.5 \mathrm{~mm} \text { anuales. La tendencia } \\
\text { señalan un aumento de la temperatura será } 1^{\circ} \mathrm{C} \text { a } 2^{\circ} \mathrm{C} 1^{\circ} \mathrm{C} \text {, con algunos cambios que } \\
\text { pueden variar en verano y en invierno. }\end{array}$} \\
\hline & $\begin{array}{l}\text { Impacto sobre los } \\
\text { recursos }\end{array}$ & \multicolumn{6}{|c|}{$\begin{array}{l}\text { Los manglares han sido afectados principalmente por disturbios naturales, en su caso, } \\
\text { vientos fuertes, avenidas extraordinarias de aguas pluviales y diversos efectos locales } \\
\text { derivados del cambio climático global. Los manglares son uno de los sistemas costeros } \\
\text { más sensibles al aumento del nivel del mar. }\end{array}$} \\
\hline Construido & $\begin{array}{l}\text { Impacto sobre la } \\
\text { infraestructura }\end{array}$ & Inundación & Caminos & Caminos & Inundación & Inundación & $\begin{array}{l}\text { No comentan } \\
\text { afectaciones }\end{array}$ \\
\hline \multirow[t]{3}{*}{ Cultural } & $\begin{array}{l}\text { Percepción de los } \\
\text { cambios ¿Perciben todos } \\
\text { los cambios? ¿Todos los } \\
\text { perciben? ¿Algunos no? } \\
\text { ¿Por qué? }\end{array}$ & $* * *$ & $* * *$ & $* * *$ & $* * *$ & $* * *$ & $* * *$ \\
\hline & $\begin{array}{l}\text { Reacción: ¿Creen que se } \\
\text { puede hacer algo? }\end{array}$ & +++ & +++ & - & +++ & +++ & +++ \\
\hline & ¿Están haciendo algo? & $===$ & $===$ & 0 & $===$ & $===$ & $===$ \\
\hline \multirow{4}{*}{ Humano } & $\begin{array}{l}\text { Conocimiento ¿Saben } \\
\text { qué hacer? }\end{array}$ & $* *$ & $* *$ & $* *$ & $* *$ & $* *$ & $* *$ \\
\hline & $\begin{array}{l}\text { ¿Reciben asistencia } \\
\text { técnica? }\end{array}$ & ++ & ++ & ++ & ++ & ++ & ++ \\
\hline & & & & & & & \\
\hline & $\begin{array}{l}\text { Tiempo / fuerza de } \\
\text { trabajo: ¿Las actividades } \\
\text { productivas les dejan } \\
\text { tiempo para hacer otras } \\
\text { acciones? }\end{array}$ & $\mu \uparrow$ & $\mu \uparrow$ & $\mu \uparrow$ & $\mu \uparrow$ & $\mu \uparrow$ & $\mu \uparrow$ \\
\hline Social & $\begin{array}{l}\text { ¿Tienen iniciativas } \\
\text { organizadas o no? }\end{array}$ & $\wedge \wedge$ & $\wedge \wedge$ & $\wedge \wedge$ & $\wedge \wedge \wedge \wedge$ & $\wedge \wedge \wedge \wedge$ & $\wedge \wedge \wedge \wedge$ \\
\hline \multirow{2}{*}{ Financiero } & $\begin{array}{l}\text { ¿Tienen recursos } \\
\text { propios? }\end{array}$ & $\$ \$$ & $\$ \$$ & $\$ \$$ & $\$ \$ \$$ & $\$ \$ \$$ & $\$ \$ \$$ \\
\hline & $\begin{array}{l}\text { ¿Pueden acceder a ayuda } \\
\text { financiera? }\end{array}$ & $+++\$$ & $+++\$$ & $+++\$$ & $+++\$$ & $+++\$$ & $+++\$$ \\
\hline Político & $\begin{array}{l}\text { ¿Tienen apoyo político? } \\
\text { ¿De qué organizaciones? }\end{array}$ & $\& \&$ & $\& \&$ & $\& \&$ & $\& \&$ & $\& \&$ & $\& \&$ \\
\hline
\end{tabular}

**: No saben qué hacer, ++: Reciben asistencia técnica, $\mu \uparrow:$ Tienen tiempo para hacer acciones, ^^^^^: Tienen iniciativas organizadas, $\wedge \wedge$ : No tienen iniciativas organizadas, ***: Perciben todos los cambios, +++: Reacción de hacer algo, __: No tienen reacción de hacer algo, ===: Están haciendo algo, 0: No están haciendo nada, $\left({ }^{\circ}\right)$ : Saben qué hacer, \$\$: tienen recursos propios, \$\$: no tienen recursos propios, +++\$: pueden acceder a financiamiento, \&\&: Reciben apoyo político de: CONAPESCA, INAPESCA, SEMARNAT, CONANP, PROFEPA, Ayuntamiento, Pronatura 
Análisis de la capacidad para ejecutar acciones de adaptación de las comunidades aledañas a la

\section{Laguna de San Ignacio}

Propuestas de acciones para la adaptación sugeridas por las comunidades aledañas a la Laguna de San Ignacio para fortalecer sus capitales y medios de vida

Las personas proponen diferentes acciones para fortalecer su capacidad de adaptación y disminuir la vulnerabilidad de los manglares y medios de vida (Cuadro IV)

Cuadro IV. Propuestas de acciones para la adaptación sugeridas por las comunidades aledañas a la Laguna de San Ignacio para fortalecer sus capitales y medios de vida, Baja California Sur, México

\begin{tabular}{|c|c|c|c|c|c|c|c|}
\hline Capitales & Comunidades & 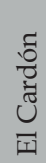 & $\begin{array}{l}\overrightarrow{\vec{T}} \\
\stackrel{\pi}{0} \\
\text { 푀 }\end{array}$ &  &  & 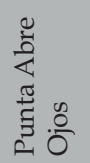 & 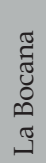 \\
\hline Capital social & & $x$ & $x$ & $x$ & $x$ & $x$ & $x$ \\
\hline Capital humano & & $x$ & & $x$ & $x$ & $x$ & $x$ \\
\hline Capital cultural & & $x$ & & $x$ & $x$ & & \\
\hline Capital político & & & & $x$ & & & \\
\hline Capital financiero & & $x$ & & $x$ & $x$ & & \\
\hline Capital físico & & $x$ & & $x$ & & & \\
\hline Capital natural & & $x$ & $x$ & $x$ & $x$ & $x$ & $x$ \\
\hline
\end{tabular}

\section{Conclusión}

La población percibe cambios en las variables ambientales de la temperatura en el agua, aire, el efecto del ENOS, y en patrones de los oleajes y las mareas. Así mismo los cambios en las variables ambientales, en sinergia con las malas prácticas de pesca y la contaminación, tienen un impacto negativo en los manglares de la LSI y en los medios de vida de las comunidades ahí asentadas. En los manglares, este impacto es mayor en el mangle blanco (Laguncularia racemosa) y en los medios de vida con mayor impacto por las variaciones climáticas y la degradación ambiental son 
la acuacultura, la extracción de la almeja de "pata de mula" y la pesca. La magnitud del impacto depende de la ubicación de los recursos: si están en zonas más calientes y frías o si están en zonas abiertas o cerradas. Las acciones que están realizando las comunidades, organizaciones e instituciones se encuentran enfocadas principalmente a fortalecer los capitales natural, económico y físico, debido a que es un área protegida y una zona económica importante para las pesquerías El trabajo relacionado con otros capitales no ha sido considerado prioritario, porque tienen más interés económicos las comunidades como El Cardón y El Dátil. Por último para la comunidad El Dátil, donde hay más conflictos internos y su capacidad adaptativa es menor, es necesario fortalecer el tejido social y la gobernanza, promoviendo la participación, buscando la cohesión social y actividades recreativas en jóvenes y niños.

\section{Literatura citada}

AVISO. 2013. Exposición al aumento del nivel del mar: tendencia de cambio del nivel del mar en el área de estudio, entre octubre 1992 y diciembre 2012. (http:/ / www.aviso.oceanobs.com/ en/news/ocean-indicators/mean-sea-level/)

BIOMARCC. 2013. Vulnerabilidad y escenarios bioclimáticos de los sistemas marino-costeros a nivel del Caribe centroamericano. Informe técnico presentado por Bouroncle, C-al Proyecto “Biodiversidad Marino Costera en Costa Rica -Desarrollo de Capacidades y Adaptación al Cambio Climático (BIOMARCC)".

Cifuentes-Jara, M. 2010. ABC del cambio climático en Mesoamérica. Primera edición, Turrialba, Costa Rica, Centro Agronómico Tropical de Investigación y Enseñanza (CATIE). Serie técnica 383.

CONABIO. 2009. Manglares de México: extensión y distribución. $2^{a}$ ed. Comisión Nacional para el Conocimiento y uso de la Biodiversidad. México. 99 pp.

DFID. 1999. Hojas Orientativas sobre los medios de vida sostenibles, Department For International Development., consultado 22 de Junio de 2013, disponible http://community.eldis. org/.59c21877/SP-GS2.pdf.

Félix Pico, E.; Serviere, Z.E.; Riosmena R. R.; León De la Cruz J.L. (Editores).2011. Los Manglares de la Península de Baja California. Centro Interdisciplinario de Ciencias Marinas-Instituto 
politécnico nacional (CICIMAR-IPN), Centro de Investigaciones Biológicas del Noroeste (CIB), Universidad Autónoma de Baja California Sur (UABCS). Primera edición, México.

Flora, C.; Flora, J.; Fey, S. 2004. Rural Communities: Legacy and Change (2nd ed.). Boulder, CO: Westview Press.

Gottret, V. 2011. El Enfoque de los Medios de Vida Sostenibles, Una Estrategia para el Diseño e Implementación de Iniciativas para la Reducción de la Pobreza, Centro Agronómico Tropical de Investigación y Enseñanza (CATIE) Turrialba, Costa Rica

Gutiérrez-Montes, I.; Siles, J. 2008. Diagnóstico de medios de vida y capitales de la comunidad de Humedales de Medio Queso. Los Chiles, Costa Rica, CATIE, UICN.

Hernández-Montilla MC, Martínez-Morales MA, Posada Vanegas G, de Jong BHJ (2016) Assessment of Hammocks (Petenes) Resilience to Sea Level Rise Due to Climate Change in Mexico. PLoS ONE 11(9): e0162637.https:/ / doi.org/10.1371/journal.pone.0162637.

IPCC. 2001. Cambio climático 2001: Impactos, adaptación y vulnerabilidad. Informe del Grupo de Trabajo II del IPCC al Tercer Informe de evaluación del Grupo Intergubernamental de Expertos sobre el Cambio Climático.

IPCC. 2007. Cambio climático 2007: Informe de síntesis. Contribución de los Grupos de trabajo I, II y III al Cuarto Informe de evaluación del Grupo Intergubernamental de Expertos sobre el Cambio.

Marshall N.A.; Marshall P.A.; Tamelander J.; Obura D.; Malleret-King D.; Cinner, J.E.2009. A Framework for Social Adaptation to Climate Change; Sustaining Tropical Coastal Communities and Industries. Gland, Switzerland, IUCN. v + 36 pp.

Mendoza-Salgado, Lechuga-Devéze, A.; Pedrín-Avilés. 2011. Capítulo 1 La calidad ambiental de los manglares de la Baja California Sur. Los manglares de la península de la baja california, edit. Félix Pico, Seviere Zaragoza, Riosmena Rodríguez y León de la Luz, Centro Interdisciplinario de Ciencias Marinas, Centro de Investigaciones Biológicas del Noroeste, S.C. y Universidad Autónoma de Baja California Sur.

PEACC-BCS. 2012. Grupos vulnerables ante los impactos del cambio climático para la Baja California Sur. Elaborado por Ángeles, Juárez, Cariño, Solís, Vázquez, Lorella, Davis, Monteforte y Amao. Plan Estatal de Acción ante el Cambio Climático para Baja California Sur, editoras Ivanova y Gámez, México. 
PEACC-BCS. 2012. Variabilidad climática: Escenarios para la Baja California Sur. Elaborado por Herrera y LLuch. Plan Estatal de Acción ante el Cambio Climático para Baja California Sur, editoras Ivanova y Gámez, México.

Ramsar. 2003. Ficha informativa de los humedales de Ramear, Laguna de San Ignacio. Consultado en: http:/ / ramsar.conanp.gob.mx/

Sandoval-Castro E, Dodd RS, Riosmena-Rodríguez R, Enríquez-Paredes LM, Tovilla-Hernández C, López-Vivas JM, et al. (2014) Post-Glacial Expansion and Population Genetic Divergence of Mangrove Species Avicennia germinans (L.) Stearn and Rhizophora mangle L. along the Mexican Coast. PLoS ONE 9(4): e93358. https:/ / doi.org/10.1371/journal.pone.0093358

Yáñez-Arancibia, A., J. W. Day, R. R. Twilley y R. H. Day. 2014. Manglares; ecosistema centinela frente al cambio climático, Golfo de México. Madera y Bosques 20(3):39-75

Yáñez-Arancibia, A.; Day, W.; Twilley, J.; Day, R.H. 2010. Los manglares frente al cambio climático ¿tropicalización global del Golfo de México? Impactos del Cambio Climático sobre la Zona Costera. Instituto de Ecología A. C. (INECOL), Texas Sea GrantProgram, Instituto Nacional de Ecología (INE-SEMARNAT), México.

Yáñez-Arancibia, A.; Twilley, R.; Lara Domínguez, A. 1988. Los ecosistemas de manglar frente al cambio climático global, Madera y Bosques, vol. 4, núm. 2, pp. 3-19, Instituto de Ecología.

\section{Cita}

Bautista-González, K.A. 2017. Vulnerabilidad de los manglares en la reserva de la biósfera el vizcaíno, e identificación de acciones de adaptación local. Áreas Naturales Protegidas Scripta, 2017. Vol. 3 (2): 93-111. https:/ / doi.org/10.18242/anpscripta.2017.03.03.02.0004

Sometido: 18 de Junio de 2017

Revisado: 25 de Agosto de 2017

Aceptado: 27 de Noviembre de 2017

Editora asociada: Dra. Magdalena Lagunas Vásquez

Idioma Inglés Abstract: Ms.C. Diana Dorantes

Diseño gráfico editorial: Lic. Gerardo Hernández 